\title{
Efficacy and Safety of Iodine-125 Brachytherapy Combined with Hepatectomy: A Meta-analysis
}

\author{
Xinyu Zhao ${ }^{1,3}$, Shaoxiong Ren ${ }^{2,3}$, Xiaopei Zhang ${ }^{1,3}$, Xiaohong $\mathrm{Wu}^{1,3}$, Aixia Sui ${ }^{3, *}$ \\ ${ }^{1}$ Graduate School of Hebei North University, Zhangjiakou 075000, China \\ ${ }^{2}$ Graduate School of Hebei Medical University, Shijiazhuang 050017, China \\ ${ }^{3}$ Department of Oncology, Hebei Provincial People's Hospital, Shijiazhuang 050051, China \\ *Correspondence Author, suiaxhebei@126.com
}

\begin{abstract}
Objective: To investigate the efficacy and safety of hepatectomy combined with iodine-125 (I-125) seed margin implantation in the treatment of hepatocellular carcinoma. Methods: The databases such as PubMed, Cochrane Library, Embase, CNKI, CBM and wanfang database were retrieved, and the clinical control study with hepatectomy combined with I-125 seed margin implantation for hepatocellular carcinoma were included. Qualified literatures were included according to the standard, and the literature quality was evaluated by the modified Jadad scale and the Newcastle-Ottawa scale. Meta-analysis was performed using RevMan 5.4 software. Results: Four studies were included, with a total of 252 patients. The results showed that there was no significant difference in 1-year survival rate between the two groups. The 3-year survival rate and 5-year survival rate of the combined treatment group were better than those of the control group. There was no significant difference in the incidence of complications between the two groups. Conclusion: Compared with simple hepatectomy, I-125 seed margin implantation assisted hepatectomy has improved clinical efficacy in the treatment of hepatocellular carcinoma without increasing the incidence of complications.
\end{abstract}

Keywords: Hepatocellular carcinoma, Hepatectomy, Iodine-125 seed, Meta analysis.

\section{Introduction}

Primary liver cancer is a common malignant tumor worldwide. In 2020, there were 910,000 new cases of liver cancer worldwide, ranking sixth among malignant tumors, and 830,000 deaths, ranking third among causes of cancer death[1], Hepatocellular carcinoma (HCC) accounts for $85 \% \sim 90 \%$ of the total[2]. Hepatocellular carcinoma is mainly treated by surgery, but the cumulative recurrence rate can reach $50 \% \sim 70 \%$ within 5 years after hepatectomy[3]. Therefore, reducing postoperative recurrence rate is the key to improve the overall efficacy of liver cancer. Iodine-125 particles continuously release gamma rays to kill tumor cells, with high irradiation dose in tumor tissues and low irradiation dose in surrounding normal organs, which is widely used in tumor therapy. Iodine-125 particle implantation combined with other therapies has a good effect on patients with primary liver cancer complicated with portal vein tumor thrombus[4, 5]. Similarly, Iodine-125 seed implantation is highly effective in patients with advanced unresectable liver cancer[6], In this paper, we collected the controlled trials of iodine-125 seed margin implantation assisted hepatocellular carcinoma resection, and conducted meta-analysis to compare the overall survival rate, non-recurrence rate and complications, hoping to provide therapeutic reference for improving the surgical prognosis of patients with HCC.

\section{Methods}

\subsection{Retrieval Strategy}

The retrieval time set in the retrieval strategy is from the database construction to August 2021. The databases searched included PubMed, Cochrane Library, Embase, CNKI, CBM and Wanfang. "Hepatocellular", "Iodine-125 Seed", "Iodine-125" and corresponding free words were used as search terms.

\subsection{Inclusion and Exclusion Criteria}

\subsubsection{Inclusion criteria}

1) Prospective and retrospective clinical controlled trials; 2) Patient: HCC has been confirmed and indicated for hepatectomy; 3) Treatment methods: Observation group: I-125 seed margin implantation combined with hepatectomy, control group: hepatectomy alone; 4) Included literature provided at least one of the following outcome measures: 1, 3, 5 -year survival, incidence of complications (abdominal infection, pleural effusion, heart disease); 5) Can provide complete case data.

\subsubsection{Exclusion criteria}

1) Review, case reports, meeting minutes, expert reviews, lectures, and repeated literature; 2) Including other treatments except I-125 margin implantation and hepatectomy; 3) Complete case data cannot be provided.

\subsection{Research Methods}

\subsubsection{Literature screening}

Two researchers independently carried out literature screening according to the inclusion and exclusion criteria formulated in advance. When differences were encountered, the third researcher joined in, and the decision was made jointly by the three researchers

\subsubsection{Quality evaluation}

The improved Jadad Scale was used to evaluate the quality of randomized controlled studies (RCTS), with a total score of 7 points and 3 points as qualified literature. The NewcastleOttawa Scale (NOS) was used to evaluate retrospective cohort studies (RCS), with 6 points as qualified literature. 


\subsubsection{Data extraction}

The extracted data mainly include: the name of the first author; Publication date; Research type; Number of cases in observation group and control group; Age; Survival rate and complication rate

\subsubsection{Statistical analysis}

All studies were randomized controlled studies using relative risk (RR) and 95\% confidence intervals; Odds ratio (OR) and 95\% confidence intervals $95 \%$ confidence intervals were used when at least one retrospective study was included. $\mathrm{I}^{2}$ value was used to measure the heterogeneity of all studies, and fixed effect model was used for $\mathrm{P} \geq 0.10, \mathrm{I}^{2}<50 \%$. When $\mathrm{P}<0.10$, $\mathrm{I}^{2} \geq 50 \%$, the random effects model is used.

\section{Results}

\subsection{Literature Retrieval Results}

Four studies were included. A total of 252 patients were enrolled, including 126 in the observation group and 126 in the control group (Figure 1).

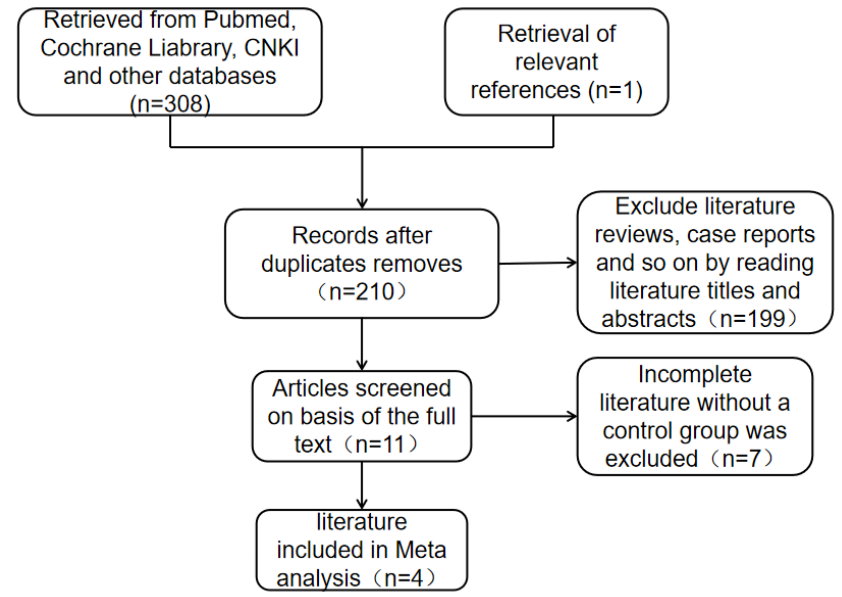

Figure 1: Literature screening process

\subsection{Basic Characteristics and Quality Evaluation}

Basic characteristics include the name of the first author; Publication date; Research type; Number of cases; Age; comparability of baseline data.4 studies were of high quality, 3 randomized controlled studies had Jadad scores of 3, 1 retrospective controlled study had NOS scores of 7 (Table 1).

Table 1: Basic Characteristics and Quality Evaluation

\begin{tabular}{|c|c|c|c|c|c|c|c|c|}
\hline \multirow{2}{*}{ The Author } & \multirow{2}{*}{ Year } & \multirow{2}{*}{$\begin{array}{c}\text { Research } \\
\text { design }\end{array}$} & \multicolumn{2}{|c|}{ Sample size } & \multicolumn{2}{|c|}{ Age(years) } & \multirow{2}{*}{$\begin{array}{c}\text { Baseline } \\
\text { comparability }\end{array}$} & \multirow{2}{*}{$\begin{array}{c}\text { Literature quality } \\
\text { score }\end{array}$} \\
\hline & & & $\mathrm{T}$ & $\mathrm{C}$ & $\mathrm{T}$ & $\mathrm{C}$ & & \\
\hline Kaiyun Chen et al[7] & 2013 & RCT & 34 & 34 & $48.91 \pm 7.30$ & $50.79 \pm 6.79$ & Yes & 3(Jadad) \\
\hline Jie Guo et al [8] & 2020 & RCT & 30 & 30 & $55.30 \pm 4.57$ & 56. $29 \pm 4.42$ & Yes & 4(Jadad) \\
\hline Xinju Lin et al [9] & 2019 & RCT & 26 & 26 & $54.80 \pm 5.40$ & $55.10 \pm 4.90$ & Yes & 4(Jadad) \\
\hline Yunfu Sun et al [10] & 2020 & RCS & 36 & 36 & - & - & Yes & $7(\mathrm{NOS})$ \\
\hline
\end{tabular}

RCT: Randomized Controlled Trial; RCS: Retrospective Control Study.

\subsection{Meta-analysis Results}

\subsubsection{Survival rates}

3 studies[7, 8, 10] reported 1-year postoperative survival, and heterogeneity tests showed no heterogeneity between studies $\left(\mathrm{P}=0.97, \mathrm{I}^{2}=0 \%\right)$. The results showed that $[\mathrm{OR}=2.37,95 \% \mathrm{CI}$ : $0.79 \sim 7.13, \quad \mathrm{P}=0.12]$, The 1-year survival rate of the observation group was slightly better than that of the control group, but the difference was not statistically significant. 2 studies [7, 10] reported 3-year postoperative survival, and heterogeneity tests showed no heterogeneity between studies $\left(\mathrm{P}=0.79, \mathrm{I}^{2}=0 \%\right)$. The results showed that $[\mathrm{R}=2.23,95 \% \mathrm{CI}$ : 1.11 4.49, $\mathrm{P}=0.02]$. 2 studies[7, 10] reported 1-year postoperative survival, and heterogeneity tests showed no heterogeneity between studies $\left(\mathrm{P}=0.95, \mathrm{I}^{2}=0 \%\right)$. The results showed that $[\mathrm{OR}=2.97,95 \% \mathrm{CI}: 1.47 \sim 5.98, \mathrm{P}=0.002]$. The 3 -year postoperative survival rate and 5-year overall survival rate of the observation group were higher than those of the control group, with statistically significant differences. The results showed that $[\mathrm{R}=2.23,95 \% \mathrm{CI}: 1.11 \sim 4.49, \mathrm{P}=0.02]$ (Figure 2-4).

\subsection{Incidence of Postoperative Complications}

3 studies[7-9] reportedthe incidence of postoperative abdominal infection, pleural effusion, and cardiac complications, and heterogeneity tests showed no heterogeneity between studies $\left(\mathrm{P}=0.30, \mathrm{I}^{2}=18 \%\right)$. The results showed that $[\mathrm{RR}=0.96,95 \% \mathrm{CI}: 0.48 \sim 1.91, \mathrm{P}=0.91]$. The difference was not statistically significant.

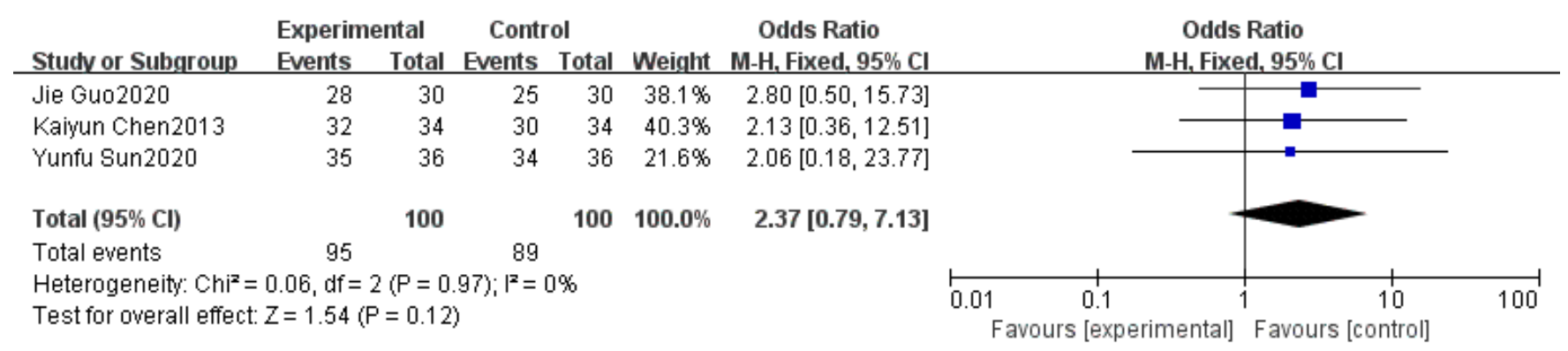

Figure 2: 1-year survival rates 


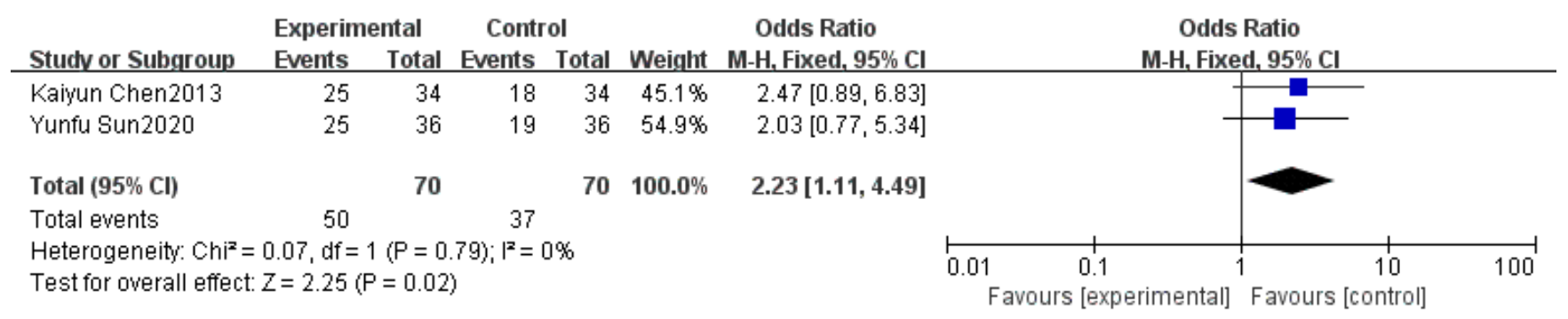

Figure 3: 3-year survival rates

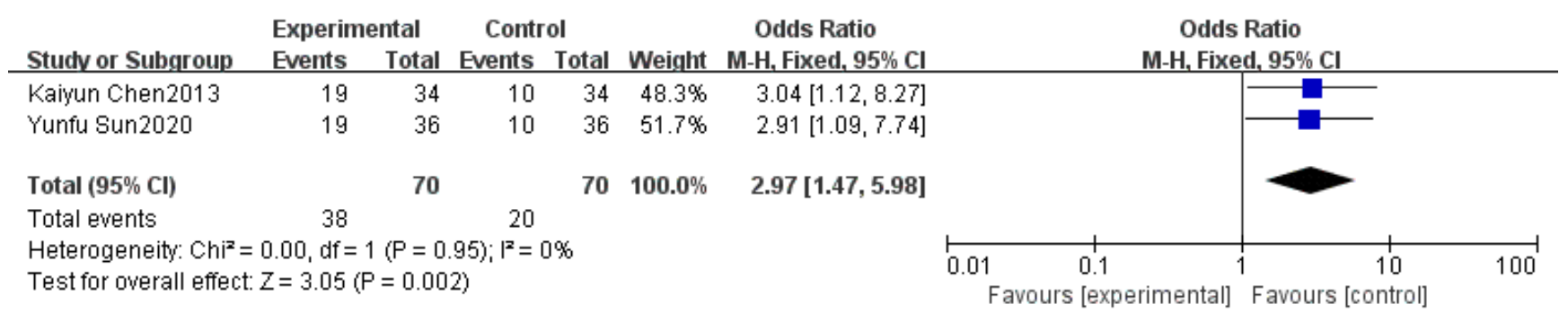

Figure 4: 5-year survival rates

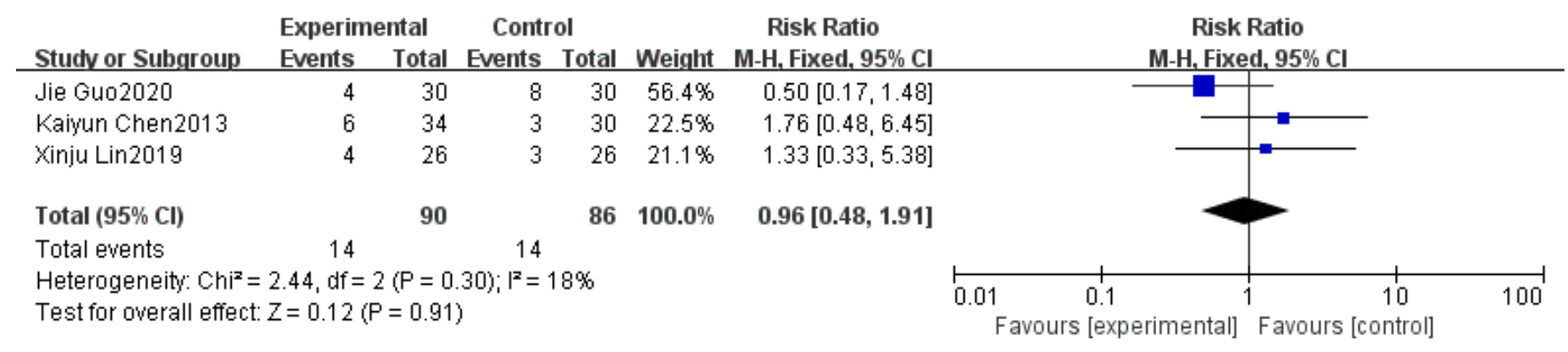

Figure 5: Incidence of postoperative complications

\section{Discussion}

At present, the treatment of liver cancer usually adopt include surgery (liver resection and liver transplantation), vascular interventional therapy, targeted, radiation and chemotherapy and so on, comprehensive treatment, liver resection is a common cancer of the liver curative measures, but liver resection patients after long-term clinical efficacy is still low, the recurrence rate is higher, multicenter recurrence and intrahepatic metastasis as the main types, reduce the postoperative recurrence rate, Improving long-term outcomes is still an important task facing modern physicians.

Iodine-125 seeds are a kind of radioactive seeds, which are implanted evenly between tumor tissues through CT or ultrasonic positioning guidance. Iodine-125 particles continuously release physical rays to kill tumor cells, which has the advantages of simple operation, little trauma and lasting effect[11], Other studies have shown that the implantation of I-125 particles can increase the number of CD3+ and CD4+ immune cells and regulate the balance of helper T cell 1 and helper T cell 2 (Th1/Th2), thus enhancing the anti-tumor immune response of patients[12]. Adverse events treated with i-125 particle brachytherapy are associated with normal tissue radiation exposure, but are usually mild and manageable. Currently, I-125 particle implantation therapy has been used in a variety of solid tumors, including prostate cancer, pancreatic cancer, lung cancer and head and neck cancer[13-16].
The results of this meta-analysis suggested that patients in the combination therapy group had a higher 1-5 year survival rate than patients in the single surgery group. The differences in 3 -year overall survival rate and 5-year overall survival rate between the two groups were statistically significant, showing good comparison ( $R R>2$ ). This is because after hepatocellular carcinoma resection, there may still be residual tumor cells, residual lesions and residual blood supply to the tumor on the resection margin tissue, so I-125 particles are implanted in the resection margin to continuously kill the remaining cancer cells and cancer tissues to achieve lower local recurrence rate and higher survival rate. There was no significant difference in the incidence of complications between the combined treatment group and the hepatectomy group, suggesting that the i-125 particle resection margin implantation assisted hepatectomy did not reduce the safety of patients. This may be because: 1) Intraoperative I-125 particle implantation was more convenient and rapid, without significantly increasing the operation time; 2) The radiation distance of I-125 particles in human tissues is short, the target radiation dose is high, and the surrounding normal tissues are less damaged by radiation.

In conclusion, iodine-125 seed margin implantation assisted hepatocellular carcinoma resection can improve the clinical efficacy of patients under the premise of ensuring good safety. However, the limitations of this study are as follows: there are few relevant studies and only a small number of cases, which cannot be grouped according to the characteristics of the margin to provide more individual guidance. Therefore, the conclusions of this study need to be confirmed by large 
sample, high quality, prospective randomized controlled trial.

\section{References}

[1] Sung H, Ferlay J, Siegel R L, et al. Global cancer statistics 2020: GLOBOCAN estimates of incidence and mortality worldwide for 36 cancers in 185 countries[J]. CA Cancer J Clin, 2021.

[2] Wan X, Du S, MAO Y. Treatment of advanced hepatocellular carcinoma in the era of comprehensive treatment[J]. Abdominal Surgery, 2021, 34(02): 92-97.

[3] Julie K H, Laura M K, Richard S F, et al. AASLD guidelines for the treatment of hepatocellular carcinoma[J]. Hepatology, 2018, 67(1).

[4] Zhang F, LI Q, Yang H, et al. Clinical value of 125I seed implantation in the treatment of primary hepatocellular carcinoma complicated with portal vein tumor thrombus [J]. Chinese Journal of Nuclear Medicine \& Molecular Imaging, 2020, 40(3): 142-146.

[5] Zhang Y, Wang T, Yue Z, et al. Efficacy of TIPS combined with $125 \mathrm{I}$ seed implantation in the treatment of hepatocellular carcinoma with portal artery thrombus and complications $[\mathrm{J}]$. Journal of Interventional Radiology, 2020, 29(1): 89-95.

[6] Li Tielian, Yang Lei, Xie Ling, et al. Clinical observation of CT-guided percutaneous implantation of 125I seeds in the treatment of advanced hepatocellular carcinoma [J]. China Medical Equipment, 2018, 15(3): 48-51

[7] Chen K, Xia Y, Wang H, et al. Adjuvant iodine-125 brachytherapy for hepatocellular carcinoma after complete hepatectomy: A randomized controlled trial[J]. PLoS One, 2013, 8(2): e57397.

[8] Guo J, Kang W, Zhang X, et al. Effect of 125I seed margin implantation on residual lesions in patients undergoing hepatocellular carcinoma resection under CT guidance [J]. Chinese Journal of clinical Oncology, 2020, 27(12): 1491-1494.

[9] Lin Xin-ju. Safety study of transect iodine-125 particle implantation in large hepatocellular carcinoma resection [J]. J Clinical Rational Drug Use, 2019,12(26):149-150

[10] Sun Y, Li W, Song Y, et al. 125I seed brachytherapy for patients with thin margin resection after hepatocellular carcinoma resection [J]. J Clin Hepatobiliary Surgery, 2012, 26(6): 426-430.

[11] Zhang Q, HAO Y. Application status of 125I radioactive seed implantation in comprehensive tumor therapy [J]. Chin J Med Sci, 2021, 11(2): 62-64, 90.

[12] Xiang G A, Chen K Y, Wang H N, et al. Immunological influence of iodine-125 implantation in patients with hepatocellular carcinoma resection[J]. Nan Fang Yi Ke Da Xue Xue Bao, 2010, 30(2): 292-294.

[13] Ito K, Saito S, Yorozu A, et al. Nationwide Japanese Prostate Cancer Outcome Study of Permanent Iodine-125 Seed Implantation (J-POPS): First analysis on survival[J]. Int J Clin Oncol, 2018, 23(6): 1148-1159.

[14] Cui S, Liu Y, Sun Q, et al. Efficacy of palliative therapy for advanced pancreatic cancer [J]. Chin J Hepatobiliary Surgery, 2017,23(04):246-250.

[15] Huang J, Yu N. Research progress of $\sim(125)$ I radioactive particle therapy for cancer $[\mathrm{J}]$. Chinese Journal of Isotopes, 2020, 33(03): 186-198.
[16] Jiang H, Yang H, Li D, et al. Treatment of non-small cell lung cancer by brachytherapy with i-125 particles [J]. Chin J Public Health, 2018, 34(12): 1684-1686. 